\title{
Homeopathic potencies vary from each other with respect to relative proportions of free and bound water molecules
}

\author{
Nirmal Chandra Sukul ${ }^{1,2,3}$, Indrani Chakraborty², Soumita Datta ${ }^{2}$, \\ Anirban Sukul ${ }^{3}$
}

\author{
(1) Department of Zoology, Visva-Bharati, Santiniketan, West Bengal, India \\ (2) Molecular Homeopathy Research Unit, Kolkata, West Bengal, India \\ (3) Sukul Institute of Homeopathic Research, Santiniketan, West Bengal, India
}

\begin{abstract}
Homeopathic potencies $12 \mathrm{CH}$ and above cross the Avogadro number, and as such do not contain any original drug molecules in their aqueous ethanol medium. It is thought $\mathrm{H}$-bonded water structures preserved by ethanol carry the information of initial drug molecules. Potentized drugs show some differences with respect to their infrared (IR) absorption spectra. In a water-ethanol solution, free water molecules vary according the concentration of ethanol. In the present study the concentration of ethanol has been kept constant at 0.03 molar fraction in 6 different homeopathic potencies. To see whether different homeopathic potencies having fixed ethanol content show variation in free water molecules, two potencies like $8 \mathrm{CH}$ and $32 \mathrm{CH}$ of three homeopathic drugs Natrum mur, Cantharis and Nux vomica were used in the study, and their ethanol concentration was kept fixed at 0.03 molar fraction. The control was considered to be aqueous ethanol at the same concentration. Spectrum of pure water was also taken. Fourier transform infrared (FTIR) absorption spectra were obtained in the wave number region of $4000-2800 \mathrm{~cm}^{-1}$. The half-width at half-maximum was measured for each spectrum. The intensity of each spectrum was normalized at $3410 \mathrm{~cm}^{-1}$ close to the peak. The difference spectrum (absorbance of drug solution - absorbance of pure water) for each drug and the control was obtained. FTIR spectra showed variation in absorbance intensity on both the high and low frequency side of the $\mathrm{O}-\mathrm{H}$ stretching band in different drugs as well as the control. The $\mathrm{C}-\mathrm{H}$ stretching band of $2977 \mathrm{~cm}^{-1}$ also showed variation in intensity in different drugs. In the difference spectra the absorbance intensity at the dip at $3630 \mathrm{~cm}^{-1}$ varied in different drugs and the control. The decrease in intensity at 3630 $\mathrm{cm}^{-1}$ and subsequent rise in intensity at lower frequency region represent the level of free water molecules and strong alcoholic $\mathrm{O}-\mathrm{H}$ band around $3250 \mathrm{~cm}^{-1}$, respectively. The drug and the control solutions show distinct variation in their FTIR spectra. The drugs have different levels of bound and free water molecules although their ethanol concentration is same.
\end{abstract}

Keywords: Homeopathic potencies, FTIR spectra, free water molecules, intensity and difference spectrum.

\section{(c)) BY-NC-ND Licensed to GIRI}

Support: Sukul Institute of Homeopathic Research.

Conflict of interest: authors declare there is no conflict of interest

Received: March 27th 2014; Revised: May 10th 2014; Published: June 30 2014.

Correspondence author: Nirmal Chandra Sukul, ncsukul@gmail.com, www.sukulhomeopathy.com

How to cite this article: Sukul NC, Chakraborty I, Datta S, Sukul A. Homeopathic Potencies vary from each other with respect to relative proportions of free and bound water moleculs. Int J High Dilution Res [online]. 2014 [cited YYYY Month dd]; 13(47):121-121. Proceedings of the XXVII GIRI Symposium; 2014 Jun 20-22; Sighisoara (Romania). GIRI; 2014; Available from: http://www.feg.unesp.br/ ojs/index.php/ijhdr/article/view/733/709 